\title{
Der Wandel von Parteien als Mitgliederorganisationen
}

Wiesendahl, Elmar: Mitgliederparteien am Ende? Eine Kritik der Niedergangsdiskussion, VS Verlag für Sozialwissenschaften, Wiesbaden 2006, 196 Seiten, $€ 29,90$.

Die Diskussionen um den postmodernen Wandel der Parteien, die Lösung ihrer gesellschaftlichen Verankerung und ihre bedrohte Funktionalität im politischen System haben in den letzten Jahren unüberschaubare Ausmaße angenommen. Die Veränderungen scheinen fundamental, in ihrem Ausgang aber so offen, dass die Prognosen so vielfältig wie wechselhaft erscheinen. Elmar Wiesendahl, bekannt für fundierte Analysen zum deutschen Parteiensystem, legt hier ein Buch vor, das die Debatten der letzten Jahre zusammenführt.

Nach einem Überblick über die wissenschaftlichen Diskussionen, bei denen sich ein Trend weg vom ,Abgesang' abzeichnet, folgt die Klärung des Begriffs ,Mitgliederpartei'. Hier setzt Wiesendahl Maßstäbe für einen feinfühligeren Umgang mit diesem Typus: Kennzeichen für ihn ist weder eine spezifische Organisationsstruktur noch die Massenbasis, sondern die Funktionalität von Mitgliedern als „strategische Organisationsressource“, die, so ihre Stärke und ihre Crux, stets gebunden ist an Mitgliedergratifikationen, welche sich in der Partizipation an programmatischen wie organisationsstrukturellen Entscheidungen als „Markenzeichen der Mitgliederparteien“ (S. 22) niederschlägt.

In Kapitel 3 folgt ein historischer Überblick der Mitgliederentwicklung, aus der Wiesendahl die Prognose ableitet, dass die Zahl der deutschen Parteimitglieder bis 2015 auf 1,25 Millionen zurückgehen wird. Das darauf folgende Kapitel bietet eine auf Statistiken der Parteizentralen fußende Empirie des Mitgliederschwunds. Als substanzielle Probleme werden das „Fehlen an Neumitgliedern“ (S. 49) und die Überalterung hervorgehoben, die auf einer "Jungmitgliederausdünnung" (S. 52) gründen, welche den abschreckenden „Altenheimcharme der Parteien " (S. 61) verstärkt. ${ }^{1}$ In Kapitel 5 stehen die vielschichtigen Gründe und Folgen des Mitgliederschwunds im europäischen Vergleich im Vordergrund. Hier finden sich die von der Parteienforschung herausgearbeiteten soziokulturellen, sozialpsychologischen und -ökonomischen Erklärungsansätze wieder. Wiesendahl steht Konzepten, die auf Anreize fokussieren, skeptisch gegenüber, während er Dealignment-Modellen, die mit Milieu-Erosion und Individualisierung argumentieren, einige Plausibilität bescheinigt. Demgegenüber würde die Deutung, kognitive Mobilisierung und postmaterialistischer Wertewandel schwächten Parteibindungen, einer empirischen Überprüfung ebenso wenig standhalten wie die "partizipatorische Verlagerungsthese“; das gleiche gelte für freizeit- und mediengesellschaftliche Ansätze. Der Zusammenhang zwischen Vertrauensschwund in die Politik und „Organisationsunlust“ der Bürger (S. 95) hingegen ist belegbar. Monokausale, modernisierungstheoretische Ansätze werden abgelehnt; stattdessen stellt der Autor ein „Erklärungspuzzle“ (S. 96) aus allgemeingesellschaftlichen Trends, zeithistorischen und regionalen Entwicklungen vor, das plausible Überlegungen, etwa zu einer stärker mentalitätsgeschichtlichen Ausrichtung der Partizipationsforschung (S. 100 f.), enthält, jedoch relativ diffus und wenig abstrakt bleibt.

Im sechsten Kapitel werden Vorschläge aus Politik und Wissenschaft vorgestellt, wie mit den Folgen gesellschaftlicher Entwurzelung umgegangen werden sollte. Die Befürworter

1 Zur Entwicklung der Parteimitgliedschaften 2006 vgl. den Beitrag von Oskar Niedermayer in diesem Heft der ZParl. 
mitgliedermarginalisierter Parteientypen gehen davon aus, dass Mitglieder in der Postmoderne dysfunktional und substituierbar seien; ob der postulierte Zusammenhang mit der Hinwendung der Parteien „zu den Massenmedien und der Staatsfinanzierung“ richtig ist, bleibe „eine empirisch noch der Bestätigung harrende Frage“ (S. 110). Wiesendahl, bekannt dafür, Mitgliederparteien als „Auslaufmodelle“ in der „Modernisierungsfalle“ zu beschreiben, schlägt sich auf die Seite derer, die Mitgliedern eine parteiorganisatorische wie gesellschaftspolitische Nichtsubstituierbarkeit bescheinigen. Auf der Basis jüngerer empirischer Forschungen, die den Fortbestand eines „traditionell organisationszentrierten Wahlkampfes" (S. 120) aufgezeigt haben, macht er deutlich, dass Mitglieder für die elektorale Funktionsfähigkeit von Parteien unverzichtbar sind. Das gilt eingeschränkt für ihre Kommunikationsleistung als bürgernahe Brückenglieder und Stimmungsbarometer. Gelungen ist auch seine Analyse, wonach entgegen der finanziellen Substitutionsthese Mitgliedsbeiträge ein „bedeutendes und unverzichtbares Standbein der Parteiengrundfinanzierung“ bleiben werden (S. 140); in der Diskussion um die Motivation von Parteieintritten zwischen Kosten-Nutzen-Abwägung und weltanschaulicher Identifikation im Zusammenhang mit der Spannung zwischen elektoraler und programmatischer Parteiorientierung bleibt Wiesendahls Position relativ undeutlich. Abschließend zeigt er die wenig erfolgreichen Bemühungen der Parteien auf, dem Mitgliederschwund durch Mitgliederwerbung, demokratisierende beziehungsweise in eine „eher pseudo-partizipatorische ... Richtung“ (S. 157) gehende Strukturreformen sowie durch die Nutzung des Internets zu begegnen.

Wiesendahls oft wirksames Vorgehen besteht darin, Forschungsbefunde und -prognosen mit gegenteiligen Studien oder Argumentationen zu konfrontieren und zu relativieren. Der Anspruch, die Debatte einer „kritischen Diskussion und empirischen Überprüfung“ (S. 13) zu unterziehen, kann dann nicht eingelöst werden, wenn, was häufig der Fall ist, allein das Fehlen empirischer Bestätigung als Gegenargument dient. Die Behauptung, Verfechter der Parteiwandelthese besäßen eine „eklatante Unkenntnis der Organisationsfähigkeit der Parteien und des tatsächlichen Wahlkampfgeschehens“, hypostasierten mediale Möglichkeiten und pflegten eine „unkritische Rezeption amerikanischer Wahlkampfliteratur“ (S. 117), erscheint überzogen.

Als Fazit bleibt ein ,Ja, aber': Mitgliederparteien drohe ein „schleichendes Ableben durch physische Auflösung“, „absehbar ist dieser Zeitpunkt [jedoch] noch lange nicht“. Mit der Catch-all-Strategie verkommen Mitglieder zum „Klotz am Bein“, dennoch läuft „die weitere Entwicklung“ allenfalls auf „Parteien mit immer weniger Mitgliedern“ (S. 172, 173, 77) hinaus.

Ärgerlich sind viele Satz- und Druckfehler (zum Beispiel S. 36, 56, 99, 120, 140, 141, $150,158,160)$, die spätestens dem Lektorat hätten auffallen müssen. Das Buch folgt dem Trend der „Abkehr von der Untergangsrhetorik“ (Heiko Biehl), ohne eine Fundamentalkritik zu bieten. Insgesamt stellt es dennoch einen Gewinn dar, weil es Forschungsansätze bündelt, Denkanstöße bietet und Perspektiven aufzeigt. Es ist auch Politikern zu empfehlen, die nicht Typen wie der „Fraktionspartei“ (Peter Radunski) oder der „professionellen Wählerpartei“ (Angelo Panebianco) das Wort reden. Wie die Rettung der Mitgliederpartei aussehen soll, bleibt offen, Wiesendahl beurteilt selbst die Aussagen seiner Kronzeugen Paul Whiteley, Patrick Seyd und Jeremy Richardson pessimistisch als „vergilbtes Vermächtnis“ (S. 178). 